\title{
Mechanical properties of concrete made from aggregate saturated in concrete interfacial agent
}

\author{
Xian $\mathrm{Li}^{1, \mathrm{a}}$, Er-jun $\mathrm{Wu}^{1, \mathrm{~b}^{*}}$, Cheng-cai $\mathrm{Li}^{1, \mathrm{c}}$, Guo-liang Liu ${ }^{1, \mathrm{~d}}$ \\ ${ }^{1}$ College of Civil and Transportation Engineering, Hohai University, Nanjing, PR China

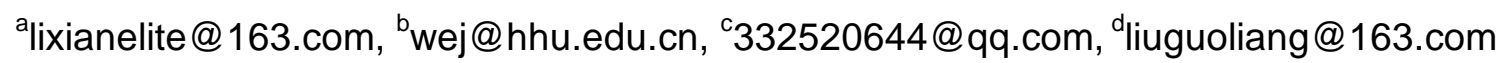

Keywords: Concrete Interfacial Agent; Modified Aggregates; Compressive Strength; Coefficient of Variation

\begin{abstract}
Based on the fact that aggregate surface features have a great effect on the performance of concrete, a method for improving concrete performance by modifying aggregate surface features was proposed. Specifically, with aggregates immersed into concrete interfacial agent (CIA) suspension to increase the bond strength of interfaces between the aggregates and mortar, mechanical properties of concrete were improved. Two group experiments were carried out and the results show that concrete with coarse aggregates saturated in CIA has higher cubic compressive strength and lower discreteness of the strength than that of conventional concrete, and cement paste grade 52.5 has a better modification result than $\mathrm{RH}-1$ paste.
\end{abstract}

\section{Introduction}

In the construction of concrete structure, reliability of the structure would increase significantly if the performance of concrete was improved, such as increasing concrete strength or lowering its discreteness. Many institutes and scholars all over the world implemented many experiments to get higher concrete performance. One of the popularity methods is adding inert filler, such as silica fume $(\mathrm{SF})$, to replace part of the cement to meet a certain requirement of design. Kwan ${ }^{[1]}$ revealed that limestone fines, fly ash and silica fume can reduce heat generation effectively and with all of them added, a cubic compressive strength can reach $65 \mathrm{MPa}$ or even higher.

While, few studies were about lowering concrete property discreteness until now . Zhang ${ }^{[2]}$ discovered that the recycled concrete made from the saturated recycled aggregate by cement paste showed a low discreteness. However, he didn't offer a method to ensure the discreteness of concrete within the standard values.

Mohamed ${ }^{[3]}$ have found that the failure of concrete was initiated from the microcracks happening in the interface between aggregate and mortar. By analyzing the failure mechanism of concrete, it is expected that deferring the occurrence and development of microcracks can improve the performance of concrete. So a new idea of applying concrete interfacial agent (CIA) to modify the coarse aggregate 
surface features was put forward. And in order to test the validity of this idea, a series of experiment was carried out.

\section{Principle and process of aggregate modified}

\section{Modified Aggregates Working Mechanism}

Before modification the concrete strength is determined by the properties of aggregate-mortar interface. However, in the concrete with modified aggregate, the previous aggregate-mortar interface (Fig 1(a), Fig 1(b)) becomes a dual-interface, as shown in the Fig 1(c) and Fig 1(d). It's well acknowledged that the bond strength of interface between CIA and aggregate is stronger than that between mortar and aggregates because of higher strength of CIA, and that the CIA and cement mortar can coalesce with each other to form a united system, whose bond strength is no less than the strength of mortar.

Coarse aggregates have varied shape and surface features. So the interfaces between aggregate and mortar show different bond strength when the concrete is loaded, which results in the high discreteness of concrete. However, after modification the aggregate surface features tend to be uniform, which can lower the discreteness in a large scale.

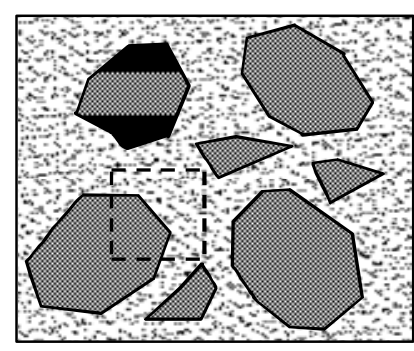

(a)

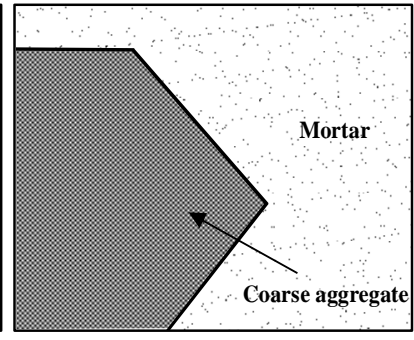

(b)

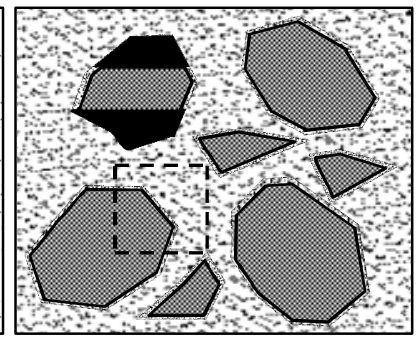

(c)

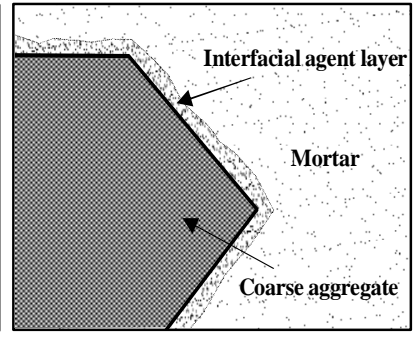

(d)

Fig 1. Sketch for the mechanism of modification: (a) (b) Macroscopic and microscopic sketch of aggregates before modification; (c) (d) Macroscopic and microscopic sketch of aggregates after modification

\section{Modifying Aggregates Process}

The main process of modification is shown as follows: First, select a kind of CIA whose bond strength is at least one grade higher than that of the cement that is the ingredient of conventional concrete. Secondly, immerse coarse aggregates into CIA suspension for 5 to 10 minutes. Finally, filter out the suspension by sieve and wait the aggregates for drying out under a cool, ventilated condition. One hour later, the modification coarse aggregates with a thin layer of CIA could be gained.

\section{Experiment}

\section{Specimen design}

The cubic specimens were classified into 11 sets, each of which contained 3 specimens. To achieve the target compressive strength of $30 \mathrm{MPa}$, the masses per cubic metre of the different 
ingredients were determined using the mix design method ${ }^{[4]}$. One set of CC mix, with $65 \mathrm{~mm}$ slump, was produced and used as reference. The rest of the 10 sets were divided into two groups evenly according to methods dealing with the aggregates, soaking or unsoaking.

Details of the concrete mix specimens are presented in Table 1. Each concrete mix specimens was assigned an identification code of M-T-X, in which M denotes the method for dealing with the coarse aggregates (soaking(S) or unsoaking $(\mathrm{U})$ ). If $\mathrm{M}$ denotes soak(S), T denotes the type of the CIA (52.5 cement (H), 52.5 cement added with CFRP (HC) and RH-1 (R)), and X denotes the CIA-water ratio. If $\mathrm{M}$ denotes unsoak(U), the labels $\mathrm{T}$ and $\mathrm{X}$ are used to identify the specimens directly mixed with the same amount of CIA adhering to the modified aggregates surfaces.

Table 1.Characteristics of test specimens

\begin{tabular}{cccc}
\hline Mix no. & Type of CIA & $\begin{array}{c}\text { Agent/Water: } \\
{[\mathrm{g} / \mathrm{g}]}\end{array}$ & Agent/Gravel: $[\mathrm{g} / \mathrm{kg}]$ \\
\hline CC & - & - & - \\
S-H-1 & 52.5 cement & 1 & - \\
S-H-2 & 52.5 cement & 2 & - \\
S-HC-1 & 52.5 cement added with CFRP & 1 & - \\
S-R-1 & RH-1 & 1 & - \\
S-R-2 & RH-1 & 2 & 1.24 \\
U-H-1 & 52.5 cement & - & 1.57 \\
U-H-2 & 52.5 cement & - & H: 1.24 C: 1.77 \\
U-HC-1 & 52.5 cement added with CFRP & - & 0.97 \\
U-R-1 & RH-1 & - & 1.2 \\
U-R-2 & RH-1 & & \\
\hline
\end{tabular}

\section{Experimental results}

The experimental results of all specimens are given in Table 2. The characteristic value of the three specimens is used for each strength result. And the coefficient of variation of the three ones is used for evaluating the discreteness out of them.

Table 2. Loading test result

\begin{tabular}{cccccccc}
\hline Mix no. & \multicolumn{2}{c}{ Compressive strength: MPa } & $\begin{array}{c}\text { Average: } \\
\text { MPa }\end{array}$ & $\begin{array}{c}\text { Standard } \\
\text { deviation }\end{array}$ & $\begin{array}{c}\text { Coefficient of } \\
\text { variation }\end{array}$ & $\begin{array}{c}\text { Characteristic } \\
\text { value: MPa }\end{array}$ \\
\cline { 2 - 4 } & 1 & 2 & 3 & & & & \\
\hline CC & 42 & 39 & 45 & 42 & 2.45 & 0.06 & 38 \\
S-H-1 & 49 & 51 & 49 & 50 & 1 & 0.02 & 48 \\
S-H-2 & 44 & 40 & 45 & 43 & 2.16 & 0.05 & 39 \\
S-HC-1 & 51 & 56 & 55 & 54 & 2.16 & 0.04 & 50 \\
S-R-1 & 44 & 48 & 46 & 46 & 1.63 & 0.04 & 43 \\
S-R-2 & 41 & 39 & 41 & 41 & 1.15 & 0.03 & 39 \\
U-H-1 & 40 & 41 & 46 & 42 & 2.64 & 0.06 & 38 \\
U-H-2 & 42 & 40 & 47 & 43 & 2.94 & 0.07 & 38 \\
U-HC-1 & 44 & 42 & 38 & 42 & 2.58 & 0.06 & 38 \\
U-R-1 & 37 & 42 & 43 & 41 & 2.64 & 0.06 & 37 \\
U-R-2 & 39 & 46 & 40 & 42 & 3.11 & 0.07 & 37 \\
\hline
\end{tabular}


From Table 2 it can be seen that the process of pre-soaking aggregates have obvious effects on the value and discreteness of compressive strength of the concrete. And the property of concrete varies when the type or concentration of the CIA changes.

\section{Parameter analysis}

\section{Effect of modification and CIA type on compressive strength}

At the end of curing period (28days), three cubes were used to determine the compressive strength of each series by using Compression Testing Machine (CTM) under pure uniaxial compression loading. The specimens U-H-1, U-H-2, U-HC-1, U-R-1 and U-R-2 were considered as the control specimens to illustrate the effect of that method on compressive strength as shown in Fig 2. The results show that the modification method can increase the compressive strength of the concrete, while the mixing of the CIA with mortar has slight influence on the strength of the specimens. That means, immersing aggregates into CIA suspension can modify concrete surface feature significantly.

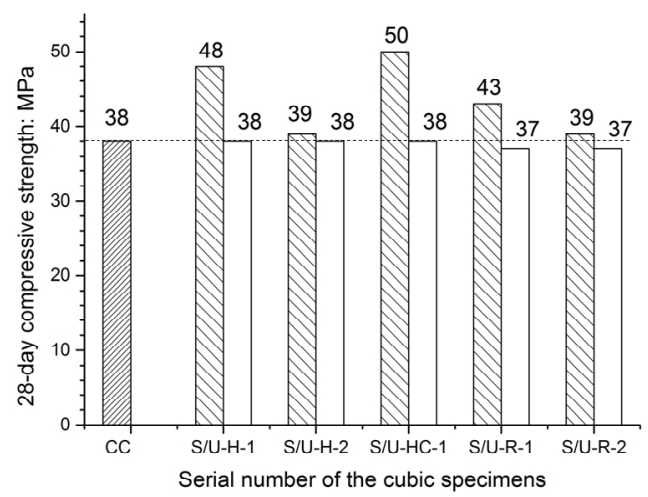

Fig 2. Compressive strength of soaking and unsoaking group

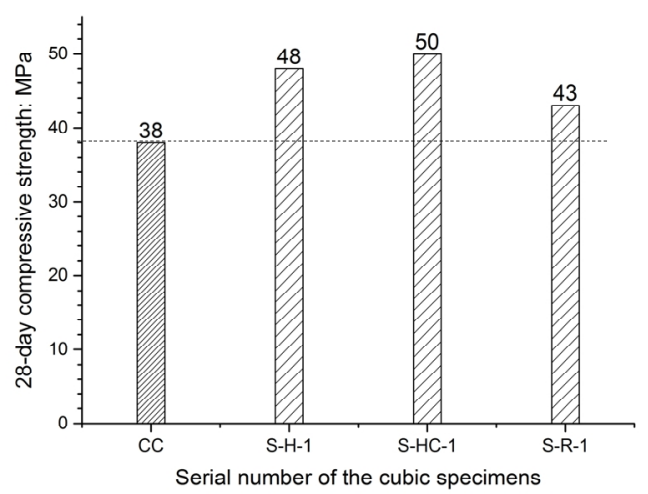

Fig 3. Influence of different CIA on compressive strength

Fig 3 shows the compressive strength of three sets improved by different types of CIA. The results show that the compressive strength was increases by $26.32 \%, 31.58 \%$ and $13.16 \%$ in specimens S-H-1, S-HC-1 and S-R-1 respectively compared to the strength of CC.

Accordingly, the adhesive ability of 52.5 cement is higher than that of $\mathrm{RH}-1$, so the effect of modification by 52.5 cement is more evident compared to that by RH-1 in same CIA concentration. Thus it's easier for 52.5 cement to reduce microcracks existing at the interface between coarse aggregate and mortar so as to increase the strength of concrete.

Through comparison between S-H-1 and S-HC-1, the addition of CFRP can promote the result of modification further. It's possibly because that the tensile strength of CFRP is much higher, so as to restrain deformation and retard the propagation of microcracks when the specimen is loaded. 


\section{Effect of methods and CIA type on discreteness of strength}

The Fig 4 shows that under the same circumstances, the two methods lead to two significantly different results. Using the aggregates modified by CIA can lower the discreteness of specimens efficiently, whereas directly mixing the same CIA with mortar has nothing to do with the reduction of discreteness. The reduction of the discreteness of specimens may be related to the changes of aggregate feature. Through immersed in the suspension, hollows of aggregates are filled by the particles and the sharp edges get blunt. So the surfaces of aggregates become smoother, which lowers the possibility of stress concentration when the specimens are loaded. And this smoothness also unifies the surface features of aggregates and finally reduces the discreteness of the concrete.

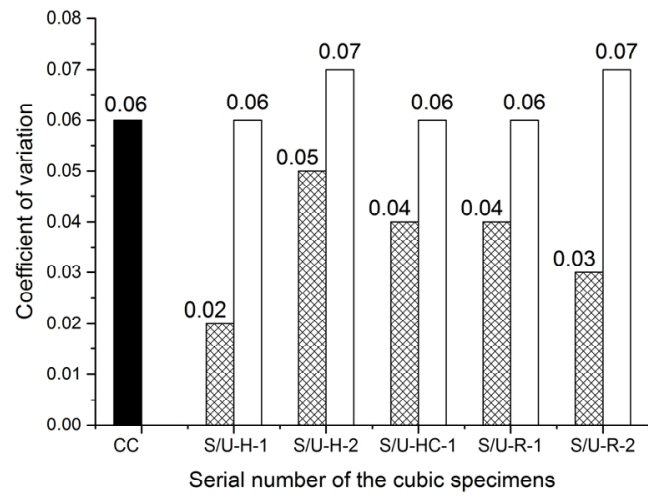

Fig 4. Coefficient of variation of soaking and unsoaking group

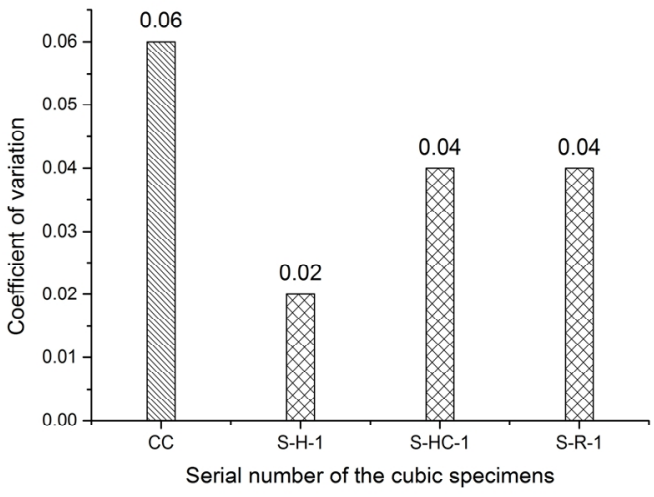

Fig 5. Influence of different CIA on coefficient of variation

Fig 5 shows the effect of CIA type on coefficient of variation. By modifying the aggregates with different CIA, the discreteness of these specimens are decreased in different scales. The discreteness of S-H-1 is lowered by $66.7 \%$; the discreteness of S-HC-1 and S-R-1 are both reduced by $33.3 \%$.

It can be indicated from Table 4 that the adhesive ability of 52.5 cement is higher than that of RH-1 because it has higher tensile strength. Therefore, at the same concentration, compared to RH-1, the aggregates immersed in 52.5 cement are wrapped more tightly by particles, the surfaces get less rough and the bond strength tends to be more uniform. Thus, the discreteness of concrete is lowered to a larger extent when the specimens are loaded.

However, when the cement paste is mixed with CFRP, the fibres are easily to intertwine and form clusters. The clusters clinging to the aggregate surfaces are distributed unevenly. The parts with more fibre clusters have higher bond strength. To put it another way, the compressive strength of specimens fluctuates in a larger range.

\section{Summary and conclusions}

The mechanical properties of the concrete containing pre-soaking coarse aggregates have been discussed above. The following conclusions can be drawn based on the experimental results under uniaxial compression loading. 
Compared to the $\mathrm{CC}$, the modification method has a great impact on the performance of concrete, specifically in increasing the compressive strength and decreasing the discreteness.

The result of modification may be better if the CIA is applied with higher bond strength: The bond strength of 52.5 cement is higher than that of RH-1. As a result, the concrete modified by 52.5 cement has higher strength and lower discreteness.

The addition of CFRP can promote the performance of concrete partially: The existence of CFRP can enhance the strength further, but it decreases the discreteness of concrete due to uneven distribution of fiber clusters.

\section{Acknowledgements}

This research is sponsored by National University Student Innovation Program (20141029403). The authors would like to thank structural laboratory of Hohai University for providing places and equipment. The authors would also like to acknowledge Miss Hu Jinlin and Mr Sun Jie for their help when conducting the experiments presented in this paper.

\section{References}

[1] Kadri EH, Duval R, Aggoun S et al. Silica Fume Effect on Hydration Heat and Compressive Strength of High-Performance Concrete. ACI Material Journal 106(2) (2009): 107-113

[2] Zhang XB, Fang Z, Deng SC Study on the Standard Deviation for the Compressive Strength of Recycled Concrete. Advances in Civil Infrastructure Engineering. 639-640(2013):313-318

[3] Mohamed AR, Hansen W Micromechanical Modeling of Crack-aggregate Interaction in Concrete Materials. Cement and Concrete Composites 21(5-6) (1999): 349-359

[4] NSCGPRC (National Standards Compilation Group of the People's Republic of China) JGJ 55-2011: Specification for Mix Proportion Design of Ordinary Concrete. NSCGPRC, Beijing, China (in Chinese) (2011). 